\title{
Mental health and decisions under risk among refugees and the public in Lebanon
}

\author{
Kai Ruggeri (10 ${ }^{1,2 凶}$, Hannes Jarke (10 ${ }^{2}$, Lama El-Zein ${ }^{3,4}$, Helen Verdeli ${ }^{5}$ \& Tomas Folke ${ }^{1,2}$
}

Lebanon is rapidly adapting public services to meet local needs as well as those of refugees from conflict regions such as Syria. However, these challenges are complicated by high volumes of individuals with poor mental health, who are also at risk of poor decision-making and may avoid the use of health services due to low trust in government institutions, among other reasons. Over 700 individuals residing in Lebanon, including Lebanese nationals, Syrian refugees and Palestinians from Lebanon, completed a series of measures covering decisionmaking with risk, mental health, and trust. The aim was to determine if significant relationships existed between these three and if those patterns were consistent between the three populations. A widely used well-being questionnaire produced similar unidimensional factor structures as found in other settings, indicating suitability for use in Lebanon, including refugees. Higher subjective well-being was associated with more risk-taking among refugees ( $\beta=0.07, \mathrm{SE}=0.02, z=4.63, p<0.01)$, but not among the Lebanese host population ( $\beta=$ $-0.003, \mathrm{SE}=0.01, z=-0.32, p=0.75)$. However, average subjective well-being did not significantly differ between the Lebanese host population and refugees (absolute difference $=$ $-1.27,95 \% \mathrm{Cl}=[-2.83,0.29]$, on a 60 -point scale), or between Syrian and Palestinian refugees (absolute difference $=-1.53,95 \% \mathrm{Cl}=[-4.16,1.08]$ ). Behavioural interventions (nudges and boosts) designed to support people in making choices more advantageous for them showed moderate effects. There is a clear pattern of greater risk-taking for refugees with better subjective well-being. This is an important finding as greater risk-taking can be associated with a number of negative health outcomes, particularly in vulnerable populations. While the behavioural interventions do show some effect on improving advantageous choice, these risk patterns are of clear interest to policymakers dealing with the health and well-being of all residents in Lebanon.

\footnotetext{
${ }^{1}$ Health Policy and Management, Columbia University Mailman School of Public Health, New York, NY, USA. ${ }^{2}$ Policy Research Group, Centre for Business Research, Judge Business School, University of Cambridge, Cambridge, UK. ${ }^{3}$ Center for Family and Community Medicine, Columbia University Irving Medical Center, New York, NY, USA. ${ }^{4}$ EmblemHealth, New York, NY, USA. ${ }^{5}$ Global Mental Health Lab, Teachers College, Columbia University, New York, NY, USA. ${ }^{凶}$ email: kai.ruggeri@columbia.edu
} 


\section{Introduction}

illions of people have been displaced and live as refugees as a result of recent conflicts in the Middle East. Lebanon currently hosts at least $1.5 \mathrm{M}$ Syrian refugees and 180,000 refugees from Palestine as of early 2019 (UN OCHA, 2019). In such a humanitarian crisis, the primary objective and concern centres on the basic needs of those affected. With policies often geared towards short-term outcomes, even in non-crisis situations (Jacobs, 2016) and policymakers often failing to anticipate future outcomes (Nair and Howlett, 2017), it is important not to overlook the impact this situation has on the decision-making of all individuals, refugee or local, living under these circumstances.

One factor directly linked to sub-optimal decision-making is poor mental health (Lawlor et al., 2020). The trauma connected to conflict situations, the resulting displacement, and ongoing stressors (Miller and Rasmussen, 2017) have negative effects on mental health (Ellis et al., 2019), which can even be passed onto future generations (Sangalang and Vang, 2017). It is also connected to the emergence of mental disorders (Morina et al., 2018). Mental health and subjective well-being (terms often used interchangeably but are distinct from mental illness) tend to be vastly underfunded and not included in cross-sectoral policies in many contexts (Jenkins et al., 2011a). However, in fragile situations, these are typically also not a policy priority, even during periods after an emergency (Jenkins et al., 2011b).

Lebanon, as a country, has taken in the highest number of refugees proportional to their population (Loesche, 2018). With mental health services being provided to Syrian refugees by Lebanese institutions, there is an opportunity to directly understand the well-being of crisis-affected, disadvantaged populations in a way that can inform health systems. This is particularly valuable given the challenges faced in the parallel system of NGOs, private practice, and UN agencies relied on by Palestinians for healthcare, who have spent protracted periods displaced in Lebanon. This is especially true for mental health and has come under tremendous pressure in recent years, to the point of extreme vulnerability or collapse (Medical Aid for Palestinians, 2020).

Life-altering events such as forced migration can impact decision-making in complex ways. On the one hand, the experience of recent and severe episodes of violence can trigger higher levels of risk aversion (Moya, 2018). On the other hand, the huge material losses people suffer from catastrophes such as natural disasters are connected to a preference for high risk-high reward options (Page et al., 2017), possibly with the aim of recovering some of what was lost, in line with prevailing theory on loss aversion (Barberis, 2013). These changes in risk preferences should not be underestimated, given the large impact they can have on individual and population-level outcomes in areas such as employment, healthcare, financial investments, addiction, and most notably, migration choices (SchildbergHörisch, 2018).

The WHO Mental Health Action Plan (WHO, 2013) suggests these context-specific challenges should be taken into account when designing national policies on mental health. One promising method that specifically fits context-dependent policy interventions is the application of behavioural insights, which is anticipated as highly valuable in Lebanon (El Chammay, 2017). Two such behavioural insights which have often shown significant effects in improving decision-making are nudges and boosts. Nudges, simple changes to the environment that influence choice without restricting any options, aim to encourage behaviours that benefit an individual (Thaler and Sunstein, 2008). Boosts, on the other hand, aim to empower individuals by strengthening their competence to achieve individually preferred outcomes (Hertwig and Grüne-Yanoff, 2017) and aim to be applicable across environments. Conflict scatters populations independent of their traits and prior status, so refugees cannot be assumed to be a homogeneous group. As such, boosting can be a particularly appealing policy strategy for supporting diverse groups such as refugees, who have highly individualised needs, and holds the potential to continue showing effects as their life situation continues to evolve. In terms of decision-making, nudges and boosts can illuminate meaningful choice patterns, as previously demonstrated in Serbia and the US (Franklin et al., 2019). The data from Serbia are particularly relevant given demographic and political parallels with Lebanon, especially in terms of population size and economics.

To assess if this approach can provide meaningful insights regarding links between mental health and decision-making in disadvantaged populations, our first step was to test the validity of a 10-item well-being measure for use in Lebanon, both for local and refugee populations. This short measure, informally referred to as a multidimensional psychological well-being scale, is commonly used in other countries for macro-level policy insights (Huppert and So, 2013; Graeff-Buhl-Nielsen et al., 2020; Ruggeri et al., 2020). It is based on the opposites of symptoms for depression and anxiety (i.e., positive characteristics) and can be used to screen differences in subjective well-being across populations (Huppert and So, 2013). We investigate differences between refugees (Syrian and Palestinian) and the Lebanese public and then explore the relation of well-being (low, average, flourishing) to risk-taking. We expand these topics to include critical contextual factors for the situation in Lebanon, primarily measures of trust, in aiming to understand links between mental health, risky choices, and the potential impact of behavioural interventions. In a final step, we test if nudges and boosts are likely to encourage advantageous choice in these populations. Described in full later, the procedure for testing this involved a series of decision-making items with varied risks and utility, which supported measuring individual ability for making optimal choices as well as general risk preferences.

We anticipated that the well-being instrument would have similar psychometric properties in Lebanon as it does in other countries, making it a good fit for linking population-level outcomes with behaviours (though it is less likely to show population-level differences between similar locations). We expected more risky choices on average for situations that were framed as losses. We further hypothesised that both interventions would increase advantageous choice, with boosts being especially effective due to their instructional nature. Finally, we expected the interventions would work by mitigating the effect of baseline risk preferences so that naturally risk-seeking individuals will be less risk-seeking in situations when risk-taking is not beneficial and vice versa.

\section{Methods}

Study design and participants. Data were collected from October to November 2019 using a professional data collection service. All aspects of the protocol were administered online in Arabic to 754 people. Lebanese participants accessed the instrument through an online portal, whereas refugees $(N=244)$ had to be approached in person due to geographic restrictions or no internet access. The total sample size exceeds the required minimum $(N=552)$ based on our a priori power calculation (see also Appendix 1: Preregistration). Based on past results in a similar context, we suspected that the demographic influences on well-being would have the lowest power, specifically $R^{2}=0.025$ for a regression model including income, age, and relationship status. An $f^{2}$-test 
suggested that 552 participants would have been required for $90 \%$ power to detect an effect of that size with an alpha threshold of 0.05 .

Participants had to be at least 18 years old, live in Lebanon, and have either Lebanese, Syrian, or Palestinian nationality. Exclusion criteria for minimum completion time were not applied as no participants failed that standard. While participants were blinded to the goals of the study, research team members who analysed the data were not. All data were collected via the online survey platform Qualtrics using a paid collection service with offices and field staff in Lebanon.

Ethical approval was granted by the Centre for Business Research, Judge Business School, University of Cambridge. Permission to collect data from vulnerable populations in Lebanon was sought and given by the Lebanese Ministry of Public Health.

Procedure and outcomes, including randomisation and masking. Participants completed 61 total items. The measures covered well-being (items provided statements such as "I have a feeling of fulfilment because of what I do", "I get help and support when I need it from people that I am close to"), decision-making, trust, and general demographics. The first 10 items assess wellbeing, followed by five measures of behaviours that predict wellbeing in wealthy countries (Centre for Well-Being and NEF, 2008). Next, all participants completed three baseline decisionmaking items before being randomly assigned by the survey platform Qualtrics to an intervention or control group. Once assigned, all participants responded to 16 additional decisionmaking items, presented in a random order varied by the participant. As participants were not aware that different interventions were tested, they were blinded to the intervention by default. Data collection partners were also blinded to group allocation during the procedure.

All decision-making items involved choosing between two financial options with risk, varied between gains and losses of differing values and probabilities. For example, at baseline participants were asked "You have \$1032. Which of the following would you prefer?" and could choose between "A 25\% chance to keep everything and a $75 \%$ chance to lose everything" or "A $100 \%$ chance to lose \$732" (loss frame), as well as "Which of the following would you prefer?" where they could choose between "A $75 \%$ chance to win $\$ 456$ and a $25 \%$ chance to win nothing" or "A $100 \%$ chance to win $\$ 300$ " (gain frame). The use of such items is the predominant method to assess risk-taking in economics and psychology (Dhar and Simonson, 2003). We refer to the option with lower probability to win but a higher potential payout as the risky choice, and to the option with a higher or certain probability of winning, but lower payout as the safe choice. Independent of whether an option is risky or safe under these terms, the choice with the higher expected value (calculated as the product of chance times potential gain) is referred to as the "advantageous choice".

Participants in the control group did not receive any form of intervention and viewed the 16 experimental items in the same format as they had seen the baseline items. Participants in both intervention groups were encouraged to pick the option with the highest expected value (the value of the outcome multiplied by its probability of occurring). The first experimental group received a disclosure nudge, which explains that the expected value is the sum of each possible outcome of the choice, weighted by the outcomes' probabilities of occurring. They were then presented with the same choices as the control group but were also presented with the expected values. These approaches have previously been tested with some success in the US and Serbia
(Franklin et al., 2019). As an example, a control group participant would see the following question:

Which of the following would you prefer?

- A $75 \%$ chance to win $\$ 303.30$ and a $25 \%$ chance to win nothing

- A $100 \%$ chance to win $\$ 200$

Participants in the disclosure condition were displayed the same item with expected values included after the options: Which of the following would you prefer?

- A $75 \%$ chance to win $\$ 303.30$ and a $25 \%$ chance to win nothing (Expected value: $\$ 227.50)$

- A $100 \%$ chance to win $\$ 200$ (Expected value: $\$ 200$ )

The second experimental group received a boost intervention. This involved a more in-depth introduction to expected values and provided instructions on how to calculate them (with a suggestion to use a calculator). Boost participants were required to accurately compute the expected values for three example sets to ensure they understood the instructions before proceeding. The full experiment with all instructions is included in Appendix 1 , including both the Arabic version and the original language in English.

For all participants, each item consisted of one certain option ( $100 \%$ chance of occurring if chosen) and one risky option (the chance of occurring if chosen is $<100 \%$ ). Items differed along four dimensions: (1) advantage (whether the risky option had a higher expected value than the certain option), (2) monetary magnitude, (3) gain or loss framing, and (4) risk (how likely or unlikely the risky option was to happen). Half the items were framed as gains, while the other half were framed as losses. Financial choice items were randomised to avoid order effects, 18 sociodemographic items were asked last to minimise confounding from stereotype threat (Nardi, 2019).

Statistical analysis. Analyses focused on validation of the wellbeing outcome measures in Arabic, modelling decision-making patterns, and assessing links between decision-making and mental health, including moderators for the three populations in Lebanon.

We conducted confirmatory factor analyses separately for the Lebanese group, Palestinian refugees from Lebanon, as well as Syrian refugees to determine the factor structure of the Multidimensional Psychological Well-Being Scale (MPWB). To determine differences in well-being between groups (Lebanese, Palestinian, Syrian), as well as to investigate how well-being relates to trust, relationship status, income, and everyday habits, we applied linear regressions. We evaluated the relationship between baseline risk-taking, well-being, and ethnicity using logistic regression. Finally, generalised linear regression models were used to quantify the relative influence of choice at baseline, nationality, and interventions on risk behaviour.

\section{Results}

Demographics. The survey commenced on 11.09.2019, and data collection concluded with the last participants on 22.11.2019. Of the 754 total participants, 510 were Lebanese, 112 were Palestinian from Lebanon, 119 were Syrian, 12 were Palestinian from Syria, and one person had another nationality. The proportion of participants was intentional, given the balance within the population in Lebanon generally as well as the challenges in accessing vulnerable Syrian and Palestinians. As the Palestinians from Syria are too small a subsample to include in the main model, but we could not assume them to be identical in traits and circumstances to another group, we excluded them from the analysis. The 
overall sample across nationalities was roughly balanced for ages and genders (see Table 1).

In the Lebanese population, more than $60 \%$ reported a university degree. Education levels in both refugee samples were much lower but equally distributed in both (see Table 1). In line with expectations, Lebanese people had both higher earnings and higher monthly expenditures than the refugee groups (for details, see Appendix Table A1), and household income correlated with expenditure for all ethnicities (Fig. 1).

Mental health in Lebanon. Using the 10 items for multidimensional well-being, each of the three countries have response distributions with means above the mid-point yet with meaningful proportions in the lower-end (see Fig. 2). This is generally in line with expectation, in that averages are only somewhat above the middle for refugee populations, and a smaller majority

\begin{tabular}{|lllll|}
\hline \multicolumn{2}{|c|}{ Table 1 Sample demographics. } & & & \\
& & & & \\
& Total & Lebanese & Syrian & Palestinian \\
\hline Participants & 741 & 510 & 119 & 112 \\
\% of sample & & 68.8 & 16.1 & 15.1 \\
Age (med) & 35 & 37 & 30 & 33 \\
$\quad$ Range & $18-69$ & $19-60$ & $18-67$ & $18-69$ \\
Female & 361 & 254 & 51 & 56 \\
& $48.7 \%$ & $49.8 \%$ & $42.9 \%$ & $50 \%$ \\
Relationship status & & & & \\
$\quad$ Single (\%) & 32.4 & 31.9 & 31.2 \\
In a relationship (\%) & & 6.5 & 1.7 & 6.2 \\
$\quad$ Married (\%) & 58.6 & 63.9 & 58 \\
$\quad$ Divorced (\%) & 2.2 & 2.5 & 1.8 \\
$\quad$ Widowed (\%) & 0.4 & Nul & 2.7 \\
Education & & & \\
$\quad$ Less than high school (\%) & & 8.2 & 73.9 & 70.5 \\
$\quad$ High school (\%) & 15.5 & 14.3 & 17.9 \\
Junior college or & 10.8 & 1.7 & 1.8 \\
Vocational training (\%) & & & \\
$\quad$ Undergraduate & 28 & 4.2 & 7.1 \\
$\quad$ degree (\%) & & & \\
$\quad$ Graduate degree (\%) & & 35.5 & 5.9 & 2.7 \\
$\quad$ Doctoral degree (\%) & 2 & Nul & Nul \\
\hline
\end{tabular}

here are experiencing the positive end of most domains. In aggregate, there are no substantive floor or ceiling effects across groups. There are only two significant group differences across all 10 dimensions, but given that is out of 30 possible contrasts, these may be spurious or noise, so we do not highlight them further.

Confirmatory factor analyses (see Appendix Table A2) indicate a good fit in all three populations (CFI $>0.9$ ) for all 10 items into a single factor. When used to compute a single factor score to reduce model complexity without reducing to a single item, those values correlate 0.99 with simple sums of raw scores. This means it is appropriate to use simple sums to compute composite scores (scale 10-70) for all individuals and groups while retaining the general model properties of a multidimensional measure. Those aggregates are normally distributed across all three groups.

We found no significant differences in average well-being between Lebanese and refugee participants (absolute difference $=$ $-1.27,95 \% \mathrm{CI}=[-2.83,0.29]$, on a 60 -point scale, $t=-1.66$, $p=0.10$ ), or between Syrian and Palestinian participants (absolute difference $=-1.53,95 \% \quad \mathrm{CI}=[-4.16,1.08], \quad t=$ $-1.18, p=0.24$ ) (see Figs. 3 and 4 ).

Risk-taking. On the whole, risk-taking at baseline did not significantly differ between nationalities. Participants took more risks in the loss-framed item relative to the other two items, though effect sizes were not extreme for Palestinians or Lebanese (see Fig. 5).

Risk-taking and subjective well-being. To examine the relationship between subjective well-being and risk-taking, we predicted risky choices at baseline from total MPWB scores, ethnicity and their interaction, with no additional covariates (for the full regression tables, see Appendix Table A3). For ethnicity, we used planned contrasts so that the first term compared Lebanese participants to the refugees and the other term compared the two refugee groups to each other. We found that higher subjective well-being was associated with higher risk-taking for the gain frame $(\beta=0.04, \mathrm{SE}=0.01, \quad z=3.70, \quad p<0.01)$ loss frame $(\beta=0.03, \mathrm{SE}=0.01, z=2.73, p<0.01)$ and neutral $(\beta=0.05$, $\mathrm{SE}=0.01, z=4.44, p<0.001)$ baseline items. The relationship between risk-taking and well-being was significantly weaker for the Lebanese nationals, relative to the refugee groups (gain: $\beta=$ $-0.02, \mathrm{SE}=0.01, z=-2.49, p<0.05$; loss: $\beta=-0.02, \mathrm{SE}=0.01$,

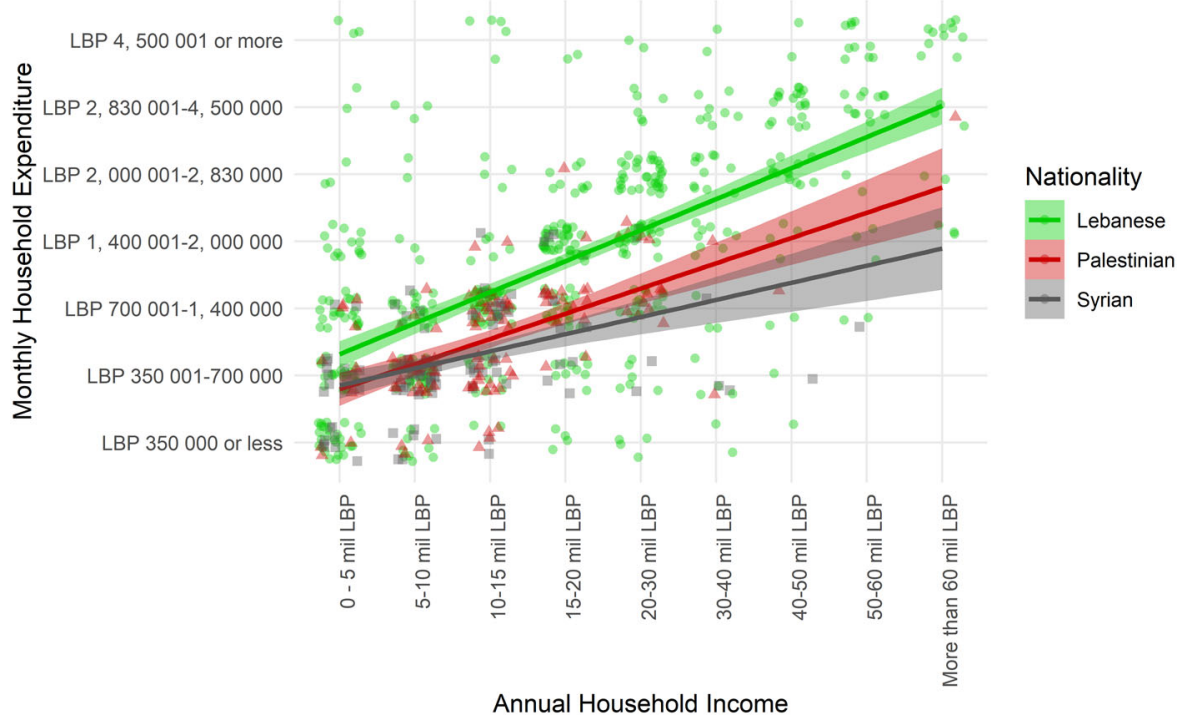

Fig. 1 Household income and expenditure. Lebanese participants have significantly higher incomes and monthly expenditure averages than Syrian and Palestinian participants across all brackets in the sample, indicated by the green line being entirely parallel to the red and black line. 


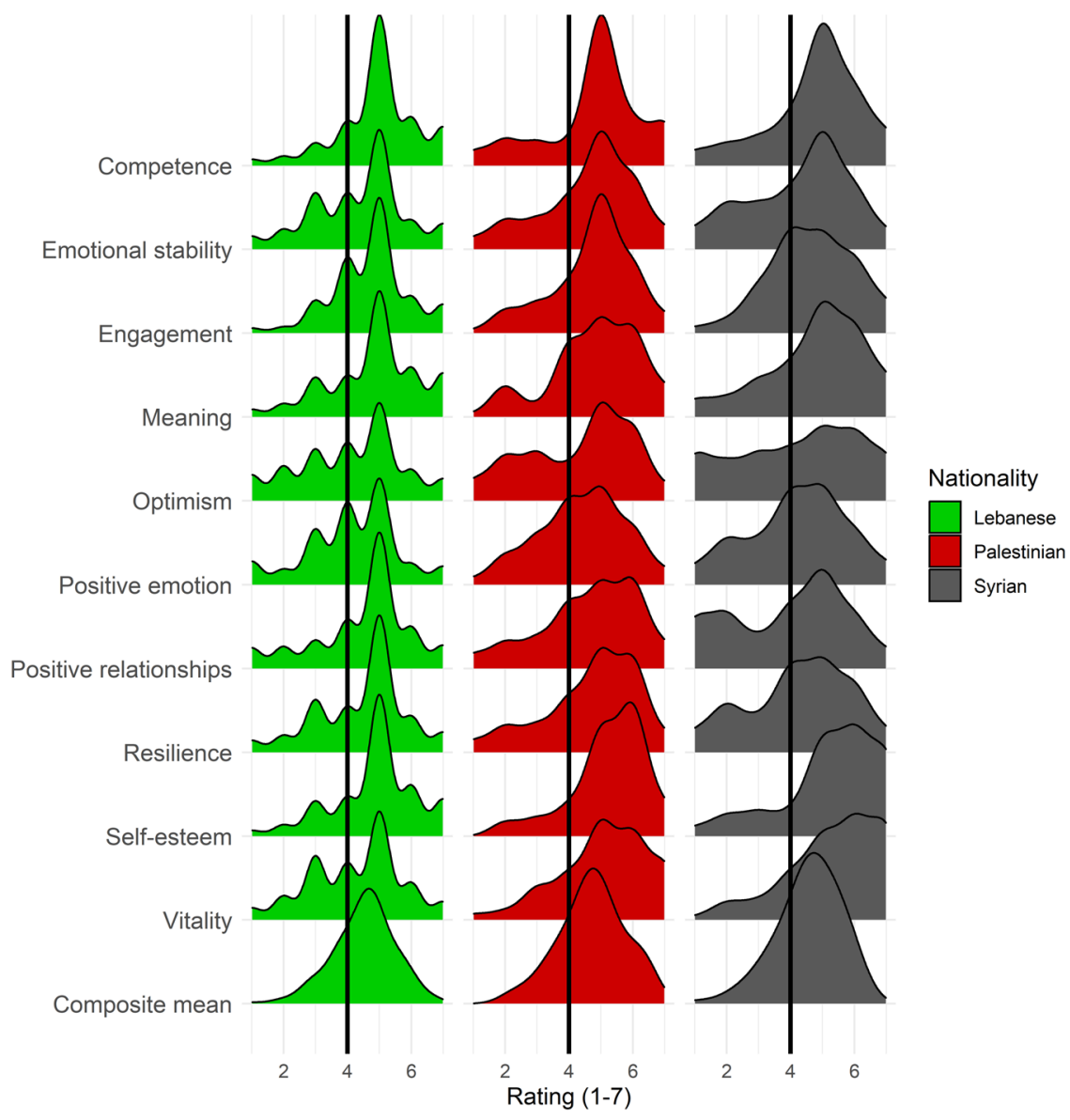

Fig. 2 Distribution of 10 well-being dimensions between Lebanese, Syrians, and Palestinians. Distributions for each individual dimension on the wellbeing measure, separated into Lebanese citizens, Palestinian refugees, and Syrian refugees. The thick line at 4 represents the scale mid-point for each item. Though distributions between countries, all means lean positive, and within-country variability is relatively stable for all three groups.

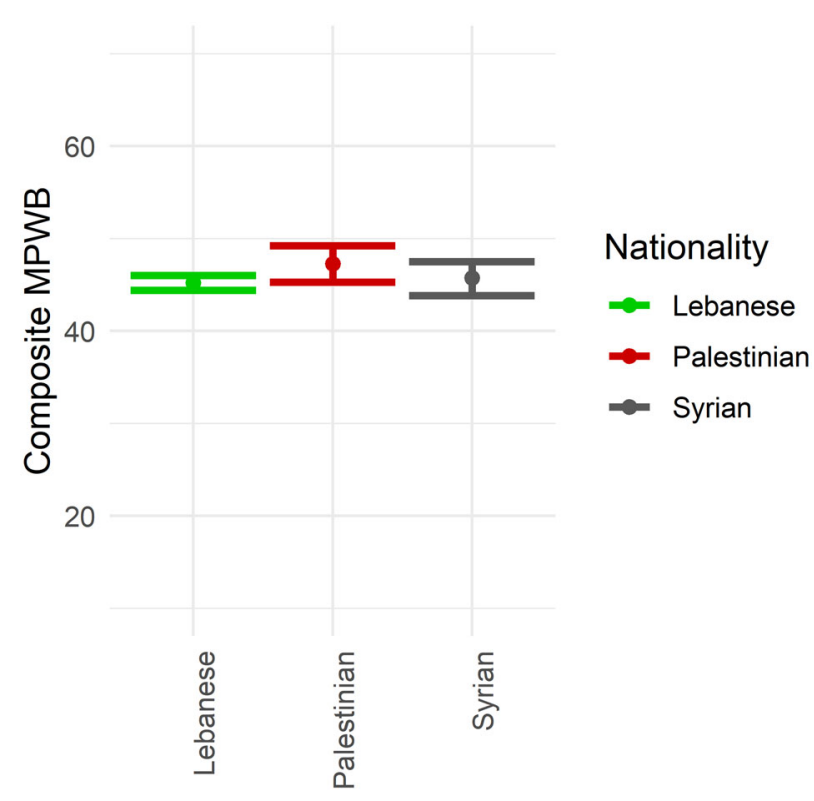

Fig. 3 Mean total MPWB by nationality, error bars show bootstrapped confidence intervals. The composite well-being scores show no significant differences between any of the three groups. The $Y$-axis represents the full range of possible values, which is presented intentionally to indicate that in spite of a wide range of possible outcomes, the three group means are generally similar. $z=-2.82, p<0.01$; neutral: $\beta=-0.03, \mathrm{SE}=0.01, z=-4.25, p<$ $0.001)$. However, the link between well-being and self-reported risk was similar across the two refugee groups. The only significant difference was for the gain item, where well-being predicted risk-taking more strongly for the Palestinians than the Syrians $(\beta=0.04, \mathrm{SE}=0.01, z=-2.54, p<0.05)$.

To visualise these relationships, we combined the two refugee groups together and binned well-being scores as poor, moderate, and flourishing. This ensured a reasonable sample size in each cell. As the overall factor structure of the well-being measure was similar, we applied the thresholds for analyses across the entire group rather than within the country (see Table 2 for within-country cutoffs). Patterns indicate clearly that the positive relationship between wellbeing and risk-taking is mostly driven by the flourishing group (Fig. 6). To see the results separated by refugee group, see Table 3.

Trust and subjective well-being. Participants generally rated their trust of others very poorly, with Palestinians indicating the lowest overall level of trust in the standard binary items (Table 4). For more granular measures, trust in family members was much higher, although Syrian and Palestinian participants trusted family members to a much greater extent than Lebanese participants. Similar patterns existed for trust in neighbours across nationalities, with greater variability in trust towards familiar people and generally low trust in strangers (Fig. 7). Using composite well-being scores and measures of trust, we find no significant relationship between the two constructs $(p=0.48)$. 


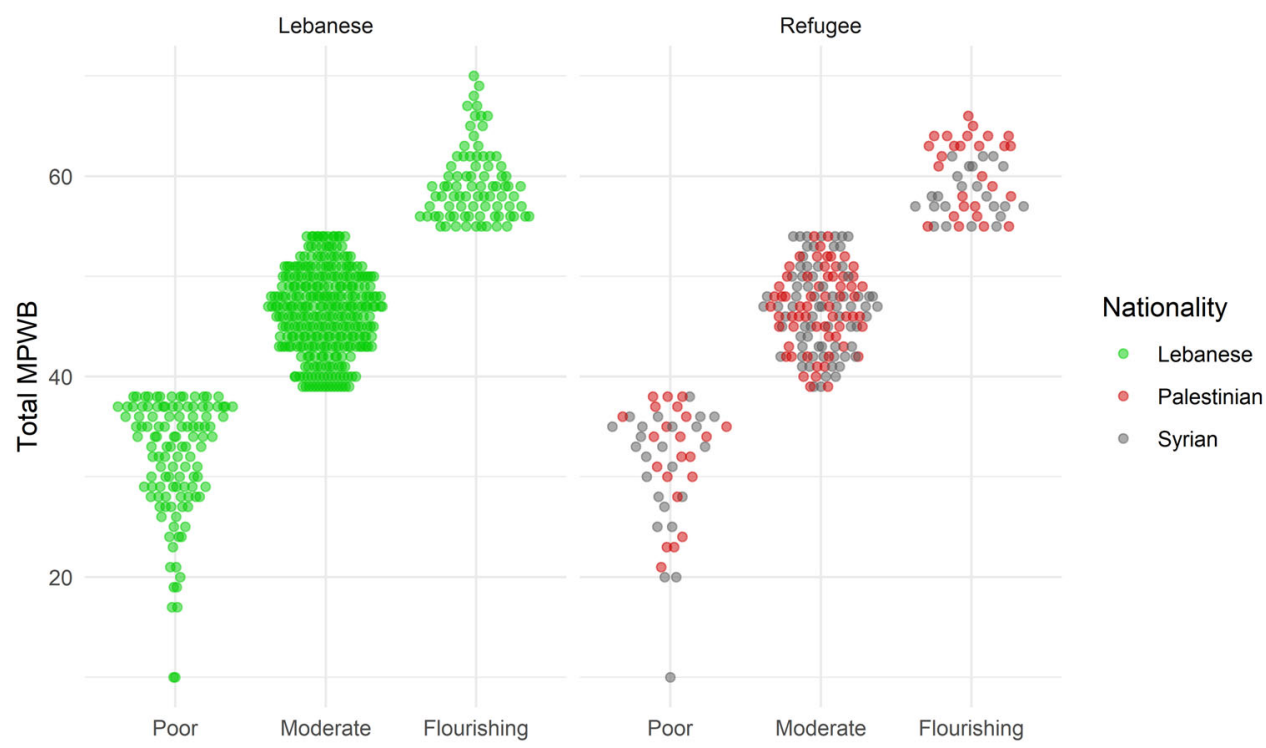

Fig. 4 Subjective well-being by nationality. Each point represents an individual participant. Groups are disaggregated based on thresholds for each mental health group (i.e., poor mental health, moderate mental health, and flourishing). The visual simply presents how the distribution is more similar between refugee and local populations in the middle, but more widely distributed between groups at the low and high ends.

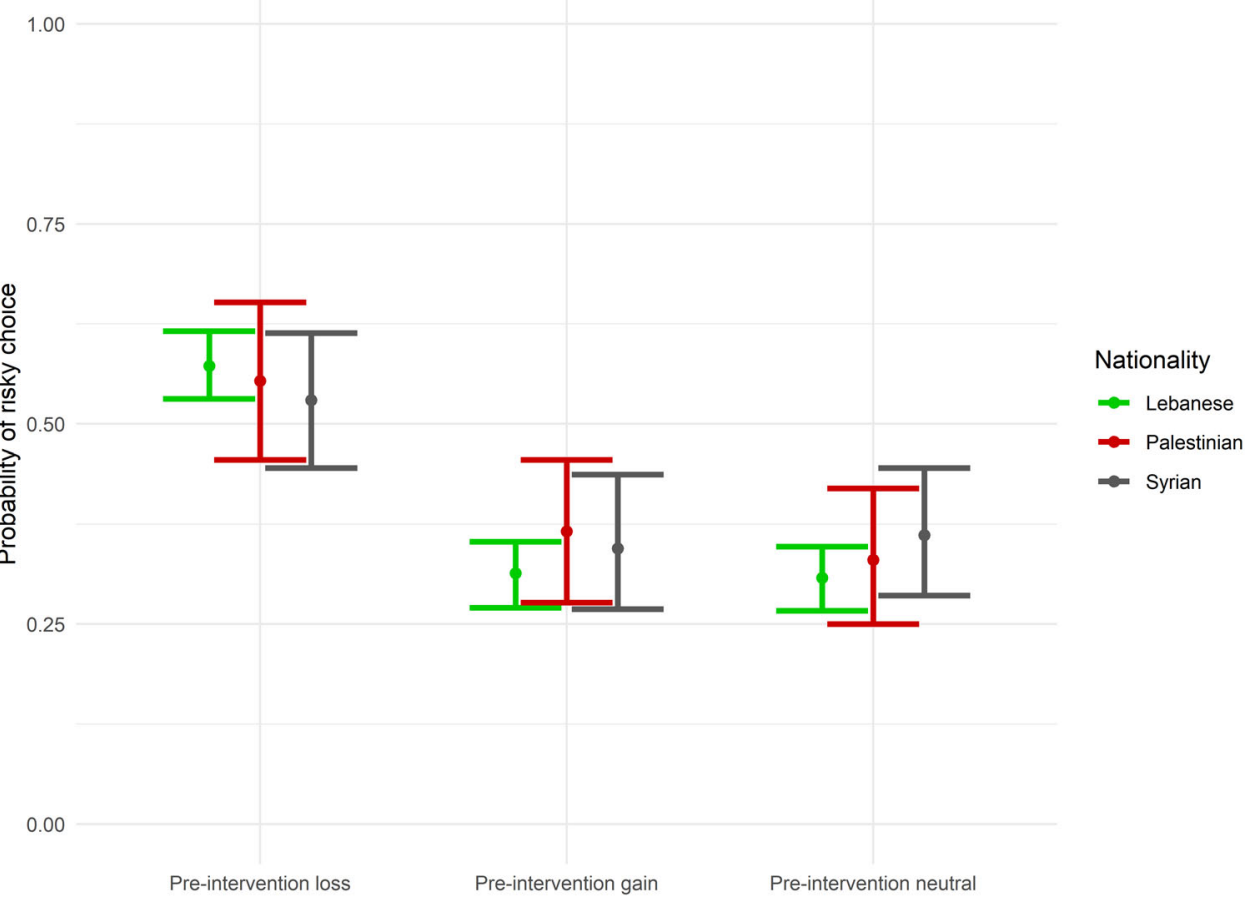

Fig. 5 Risky choice proportion at baseline. For all three populations, the probability of risky choice was higher in the group where the pre-intervention item was loss-framed.

Risk-taking and trust. We predicted risk-taking at baseline from trust in Lebanese citizens and refugees using logistic regression. We predicted risky choices at baseline from total trust scores, ethnicity, and the interaction of the two, with no additional covariates. Lebanese participants that report being more trusting are slightly more risk-seeking for the Baseline Gain Frame item $(\beta=0.05, \mathrm{SE}=0.02, z=2.18, p<0.05)$. Otherwise, trust does not predict risk propensity for any nationality for any item. For full regression tables, see Appendix Table A4.

\section{Intervention effects}

To examine how the interventions and baseline choices impacted the post-intervention decision-making, we ran linear models predicting advantageous choice (whereby the advantageous choice is the one with the higher expected value) scores in the gain frame and loss frame separately, for each of the three nationalities (Lebanese, Syrian, and Palestinians). Participants were assigned randomly to an intervention or the control group, with the number of participants in the boost group $(n=187)$ 
Table 2 Well-being scores by country.

\begin{tabular}{|c|c|c|c|c|c|c|c|c|c|}
\hline & & \multicolumn{2}{|c|}{ Lebanese } & \multicolumn{2}{|l|}{ Syrian } & \multicolumn{2}{|c|}{ Palestinian } & \multicolumn{2}{|l|}{ All } \\
\hline Range & & 10 & 70 & 10 & 62 & 21 & 66 & 10 & 70 \\
\hline \multicolumn{10}{|l|}{ Well-being group ranges } \\
\hline Poor mental health & Lower $20 \%$ & $10-37$ & & $10-38$ & & $21-39$ & & $10-38$ & \\
\hline Moderate mental health & Middle $60 \%$ & $38-53$ & & $39-54$ & & $40-55$ & & $39-54$ & \\
\hline
\end{tabular}

Groups indicate the minimum composite score to be considered to have poor mental health (bottom $20 \%$ within group), moderate mental health (middle $60 \%$ of group, between 20 th and 80 th percentile), or flourishing (highest $20 \%$ of group).

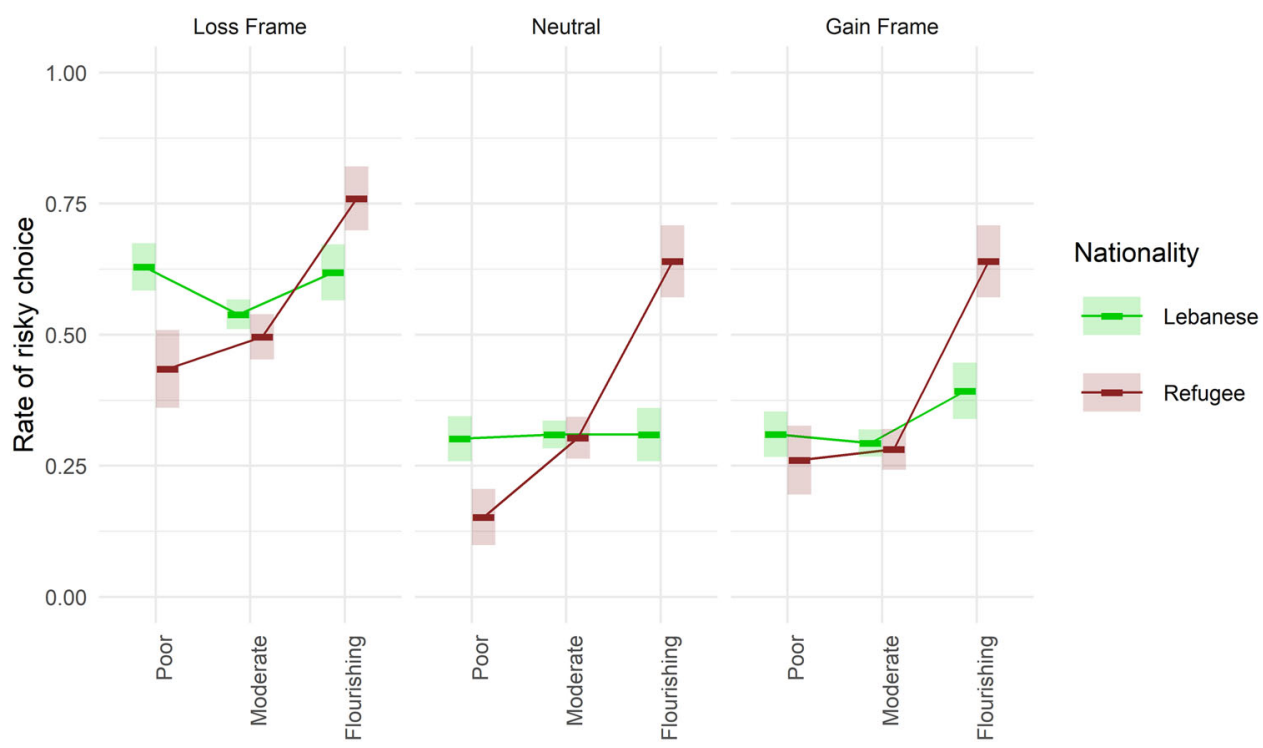

Fig. 6 Risk behaviour by subjective well-being. Thick lines represent group means. Shaded areas represent 95\% bootstrapped confidence intervals.

\begin{tabular}{|lllll|}
\hline \multirow{2}{*}{$\begin{array}{l}\text { Table } 3 \text { Percentage of participants choosing the risky option } \\
\text { at baseline by nationality and mental health. }\end{array}$} \\
Baseline item & Nationality & \multicolumn{3}{l}{ Mental health } \\
\cline { 3 - 5 } & & Poor & Moderate & Flourishing \\
\hline Gain frame & Combined & 29.6 & 29.0 & 48.5 \\
& Lebanese & 31.0 & 29.4 & 39.3 \\
& Palestinian & 31.8 & 27.0 & 63.0 \\
\multirow{4}{*}{ Loss frame } & Syrian & 20.8 & 29.2 & 65.2 \\
& Combined & 57.4 & 52.6 & 67.2 \\
& Lebanese & 62.9 & 53.9 & 61.9 \\
\multirow{3}{*}{ Neutral } & Palestinian & 31.8 & 52.4 & 81.5 \\
& Syrian & 54.2 & 47.2 & 69.6 \\
& Combined & 25.9 & 30.8 & 43.3 \\
& Lebanese & 30.2 & 31.0 & 31.0 \\
& Palestinian & 13.6 & 23.8 & 70.4 \\
& Syrian & 16.7 & 36.1 & 56.5 \\
\hline
\end{tabular}

being smaller than in the nudge $(n=287)$ or control group ( $n=276$, see also Appendix Fig. A1). This randomisation appears to have been successful in that the different intervention groups do not differ along the demographic characteristics we measured within their respective nationalities (see Appendix Table A5).

For the Lebanese, the boost condition was associated with more advantageous choices than the control condition in both the gain

\begin{tabular}{|lllc|}
\hline \multicolumn{2}{l}{ Table $\mathbf{4}$ General trust by nationality. } & & \\
& Lebanese & Syrian & Palestinian \\
\hline Most people can be trusted (\%) & 12.4 & 13.4 & 6.2 \\
Need to be very careful (\%) & 87.6 & 86.6 & 93.8 \\
\hline
\end{tabular}

frame $(\beta=0.86,95 \% \mathrm{CI}=[0.5-1.22], t=4.75, p<0.01)$ and the loss frame $(\beta=0.75,95 \% \mathrm{CI}=[0.33-1.17], t=3.62, p<0.01)$. The interventions did not improve decision-making for the refugee populations. Baseline choices did not consistently predict experimental choice in either frame, nor did they consistently moderate the effectiveness of the interventions.

Though baseline choice did not directly impact advantageous choice in the gain frame $(\beta=0.25,95 \% \mathrm{CI}=[-0.15-0.65]$, $t=1.27, p=0.21)$ nor the loss frame $(\beta=0.00,95 \% \mathrm{CI}=[-0.36$ to 0.36$], t=-0.01, p=0.98)$, it did interact with the boost intervention in the loss frame, so that participants who made advantageous choices baseline benefitted most from the intervention $(\beta=0.61,95 \% \mathrm{CI}=[0.01-1.21], t=2.00, p<0.05)$. The disclosure condition was not associated with more advantageous choices than the control condition for either frame, nor did it interact with baseline choice. The same pattern existed for the gain frame, though not the effect is not significant $(\beta=0.61,95 \%$ $\mathrm{CI}=[-0.03$ to 1.25$], t=1.87, p=0.06)$. In the Syrian sample, 


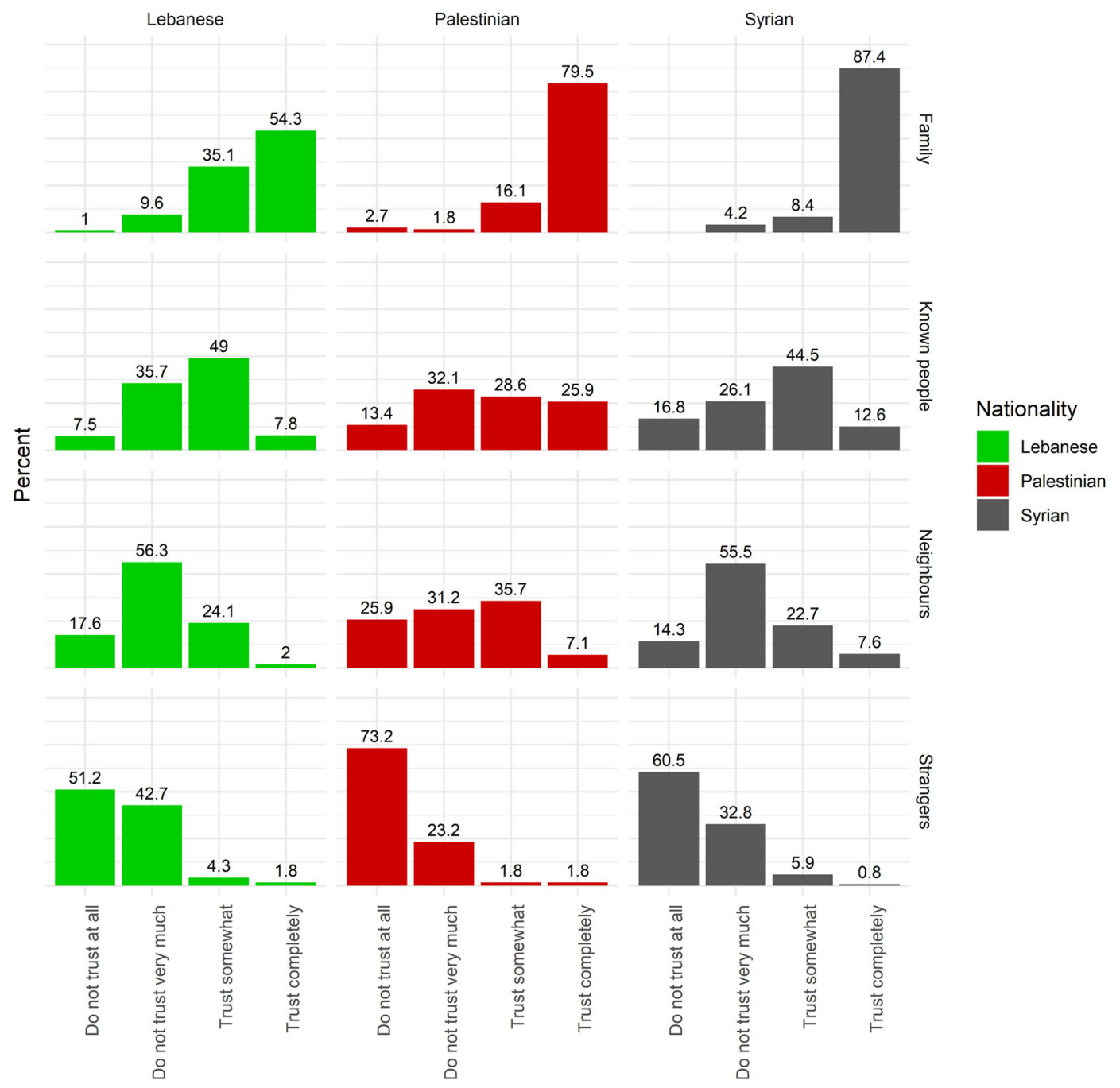

Fig. 7 Trust by nationality and domain. Bars represent response proportions for each trust domain.

none of the interventions significantly impacted advantageous choice, either in the gain frame or the loss frame.

Baseline choices did not predict experimental choices, nor did they moderate intervention effectiveness in either frame. We had similar null-findings for Palestinians from Lebanon for the gain frame. However, for the loss frame, the disclosure nudge was associated with significantly less advantageous choices than the control condition $(\beta=-0.88,95 \% \mathrm{CI}=[-1.44$ to -0.32$], t=$ $-3.17, p<0.01)$. Participants who picked the advantageous option at baseline were less negatively impacted by the disclosure intervention. However, this effect was not significant $(\beta=0.72$, $95 \% \mathrm{CI}=[-0.08$ to 1.52$], t=1.76, p=0.08)$.

To test if the effect of the conditions differed significantly between the different nationalities, we ran additional models on the full sample, predicting advantageous choice from nationality (treating Lebanese as baseline), baseline decision-making, the interventions and their interactions (but again keeping choices in the gain frame and loss frame separate). As expected from the prior analyses, boosts in the gain frame were significantly more effective for the Lebanese participants than for the Syrian $(\beta=$ $-1.13,95 \% \mathrm{CI}=[-1.87$ to -0.39$], t=-3.08, p<0.01)$ or Palestinian participants $(\beta=-0.89,95 \% \mathrm{CI}=[-1.67$ to -0.11$]$, $t=-2.26, p<0.05)$. The same pattern held for the loss frame (Syrian: $\beta=-0.83,95 \% \mathrm{CI}=[-1.65$ to -0.01$], t=-2.00, p<$ 0.05 ; Palestinian: $\beta=-0.94,95 \% \mathrm{CI}=[-1.76$ to -0.12$], t=$ $-2.27, p<0.05)$. Finally, in the loss frame, the disclosure nudge led to significantly fewer advantageous choices for the Palestinian participants relative to the Lebanese participants $(\beta=-1.08,95 \%$ $\mathrm{CI}=[-1.88$ to -0.28$], t=-2.70, p<0.01)$. See Fig. 8 for a visualisation of these results.

\section{Discussion}

Summary. In this study, we find a number of relevant insights, most notably the clear difference in risk-taking for refugees based on mental health. The 10-item well-being measure used is especially valuable in this context: it includes dimensions such as vitality, resilience, and positive relationships. Each of these is established as critical to the well-being of vulnerable populations.

That individuals higher on those dimensions in aggregate demonstrate a higher willingness to take risks prior to any intervention may indicate a greater openness to seek opportunities to improve their current circumstances without extensive deliberation over likely outcomes. It may also have a more positive interpretation, such as being more willing to seek care or other support in situations of extreme duress or even to take proactive steps to improve their financial circumstances. These findings should be replicated and explored further, ideally in more precisely-defined and ecologically-valid scenarios, before drawing any absolute conclusions, though.

There is no single application of the risk-flourishing insight to vulnerable populations. Instead, it broadly highlights the importance 

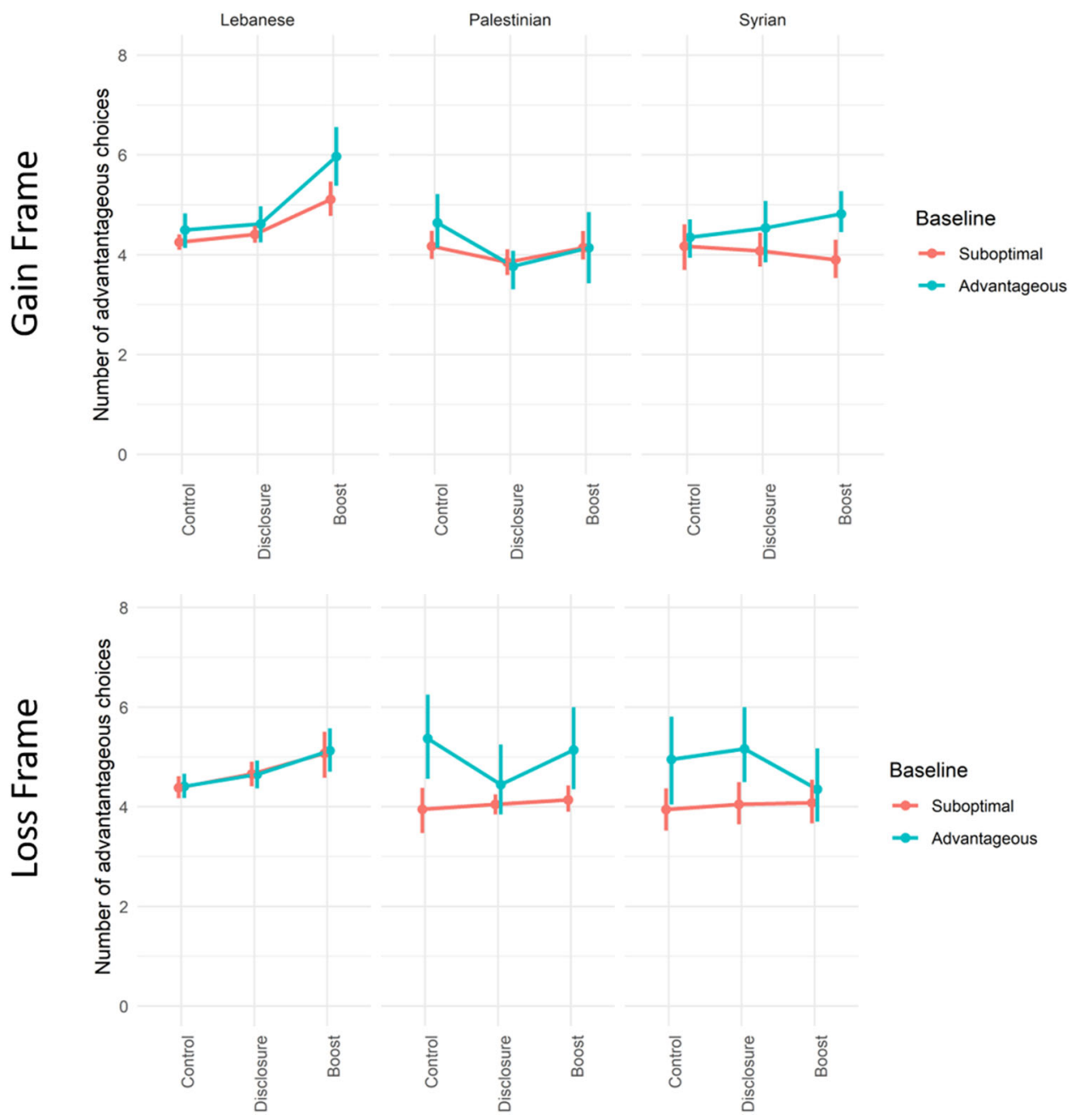

Fig. 8 Advantageous choices by nationality and intervention group. Codes for the baseline choice congruent with the frame of the experimental items. Error bars are $95 \%$ bootstrapped $\mathrm{Cl}$. Orange indicates those individuals who made a suboptimal choice at baseline; turquoise represents those who made an advantageous choice at baseline.

for health systems to not limit consideration to individuals with poor mental health. While poor mental health has long been associated with a number of risk factors such as alcohol and drug abuse, our findings indicate those with better mental health in displaced populations may produce another sort of risk not typically factored into healthcare reforms. Experiencing recent and severe episodes of violence is highly associated with high levels of anxiety (Moya, 2018). It should be kept in mind that the quality of risk-averse behaviour associated with high anxiety may be of a more holistic quality, affecting more behaviours than economic decisions possibly including the choice to seek help. This can prove especially problematic in a population at high risk of developing clinical symptoms due to trauma and even passing these on to their children (Santavirta et al., 2018).

We do not have data to infer how specific or global this risk preference is, nor how it may impact health, such as delaying care. Unfortunately, we also lack data to infer the extent of potential risk-taking, such as having a propensity to undertake high-risk journeys to locations further away, resort to high-risk financial options (e.g., gambling), or greater likelihood to fall victim to scams. Such data would be invaluable from future study, particularly if it can be matched with our nascent findings on effective interventions, which would directly inform policy actions.
Another noteworthy finding is the general similarity in mental health patterns between local and displaced populations in Lebanon. It is too far to infer directly that this negates the need for parallel healthcare systems that provide discrete services for locals and refugees, as has been used for Palestinians. Apart from the issue of separate funding sources for refugee healthcare and healthcare for Lebanese nationals (Medical Aid for Palestinians, 2020), the low trust we observed in refugees may also hinder uptake of mental health service, if offered. However, such information may be useful to explore further as it may offer insight into aspects of mental health services that could overlap between those populations.

With regards to interventions targeting financial risk-taking, we found that boosts were the most effective intervention for Lebanese participants, regardless of whether the aim of the choice was to maximise gains or minimise losses. This suggests that boosts, and other interventions designed to support people to make autonomous decisions, might be particularly effective in middle-income countries. While previous research in Serbia employing the same methodology found that boosts were generally more effective for people who made more suboptimal choices initially (Franklin et al., 2019), we find this only to be the case for Lebanese nationals in the loss condition. 
Limitations. The well-being measure has the benefit of being multi-faceted, but each specific domain is tested in a single item format. With regards to risk-taking, while hypothetical financial choices ('gambles') are common in behavioural research, they cannot fully approximate risk preferences in real-world contexts, particularly those relating to health behaviours or other extremely stressful, uncertain situations. As such, their generalisability to risk behaviours most relevant to a refugee population may be limited. Some group differences may also be influenced by different testing environments, as the refugee population was tested in-person rather than remotely. However, we expect this to mostly impact items with a strong social desirability component (see Richman et al., 1999; Krumpal, 2013) and therefore should not have impacted our main findings. While this could potentially include well-being, it would be rather speculative to assume significant bias in either direction, as an effect in the other direction (e.g., through role confirmation: refugees who have a negative image of refugees) cannot be ruled out with complete certainty either.

Another limitation to consider is that it is possible that the observed association between risk-propensity and well-being is not driven by refugee status per se but rather other socioeconomic, legal, structural, or cultural factors that covary with refugee status. The randomisation process appears to have been effective at balancing groups across demographic background between the experimental conditions to test those aspects, but we lack the sample size to investigate these complex relationships in much depth. The only difference between the experimental conditions was based on the local population having more years of education on average, which applies across the study. However, we encourage future research comparing the risk-behaviour of refugees to well-matched controls as this would have implications for intervention design.

Further research could provide a wider range of risk domains, and if it relates to healthcare, include choices regarding general behaviours (e.g., diet, exercise, tobacco use) and those related to system use (e.g., preventive care, vaccination, delaying treatment).

It is also important to note that neither intervention worked for the refugee groups. This might be the result of different modalities of presentation (e.g. online versus face to face) but might also suggest that vulnerable populations may require more robust decision-support. Further, we cannot assess if expected values were fully understood, especially in light of differences in education level between the groups. With the majority of refugees reporting their education to be less than high school, it is possible that many may not have been familiar with the concept of probability or multiplication, which would make it hard to understand the concept of expected values. Additional work is needed to establish which one of these explanations is correct. While we did not test this directly, there is evidence (Cohn et al., 2015) to corroborate the finding that poorer mental health (at least in terms of heightened stress levels) can reduce risk-taking.

While there is no specific recommendation for what implications to infer from it, the three populations are potentially very different, even between the two refugee groups. For this reason, we presented all analyses with extensive descriptive modelling with groups disaggregated. While this does create some complexity in the analyses and visualisations, we wanted to avoid masking any potential group differences. Finally, the authors believe it is important to note that the entirety of this research was conducted prior to the unfortunate explosion in Beirut in August 2020. All data had been collected and analysed, and most writing already completed. Work also mostly predated the unfortunate government and economic circumstances that worsened during the coronavirus pandemic. Readers may wish to factor in those impacts in their review of our findings and conclusions.

\section{Conclusion}

The decision to flee your home country in times of conflictwhether alone, with children, or in a group-is perhaps the most extreme example of a choice made under risk and uncertainty. It is taken under severe duress, and, for many without strong connections outside of their home country, it may appear an equally risky option as facing the dangers at home, encouraging them to remain. The resulting impact on those choices and the ability to persevere despite potentially dire circumstances can have a lasting impact on individuals, families, and populations. That the destination country, in this case, Lebanon, has gone through substantial change over the past three decades, not least the 2005 withdrawal of Syrian troops after a 29 -year presence, has resulted in local challenges to economic stability while testing population resilience.

To better understand these impacts, we measured well-being and risk-taking in refugee and host populations. Our finding that refugee populations have similar well-being profiles to the host population may imply that the Lebanese systems have been unusually successful or that resilience among Syrians and Palestinians has been remarkably high (or a combination thereof). However, those conclusions need to be directly assessed, and our findings need to be independently replicated. The relationship between risk-taking and well-being in refugee populations has, to the best of our knowledge, never been reported before and if mapped to a broader range of risky behaviours may provide key guidance to policymakers who try to support these vulnerable groups. Responding effectively would provide added security to the health and well-being of some of the world's most vulnerable populations.

\section{Data availability}

All data and materials, including the pre-registration, are available on the Open Science Framework via https://osf.io/f2rck/ (https://doi.org/10.17605/osf.io/f2rck).

Received: 22 October 2020; Accepted: 12 April 2021; Published online: 11 May 2021

\section{References}

Barberis NC (2013) Thirty years of prospect theory in economics: a review and assessment. J Econ Perspect 27:173-196

Centre for Well-Being, NEF (The New Economics Foundation) (2008) A report presented to the Foresight Project on communicating the evidence base for improving people's well-being. Centre for Well-Being, NEF (The New Economics Foundation).

Cohn A, Engelmann J, Fehr E, Maréchal MA (2015) Evidence for countercyclical risk aversion: an experiment with financial professionals. Am Econ Rev 105:860-885

Dhar R, Simonson I (2003) The effect of forced choice on choice. J Mark Res 40:146-160

El Chammay R (2017) A policy implementer's perspective. World Psychiatry $16: 43-44$

Ellis BH, Winer JP, Murray K, Barrett C (2019) Understanding the mental health of refugees: trauma, stress, and the cultural context. In: Parekh R, Trinh NT (eds) The Massachusetts General Hospital textbook on diversity and cultural sensitivity in mental health. Springer Nature, Switzerland AG, Cham, pp. 253-273

Franklin M, Folke T, Ruggeri K (2019) Optimising nudges and boosts for financial decisions under uncertainty. Palgrave Commun 5:1-13

Graeff-Buhl-Nielsen S, Garcia-Garzon E, Benzerga A, Folke T, Ruggeri K(2020) Global mental health: an improved measure of well-being in multiple languages. Health Qual Life Outcomes 18(1):1-14

Hertwig R, Grüne-Yanoff T (2017) Nudging and boosting: steering or empowering good decisions. Perspect Psychol Sci 12:973-86

Huppert FA, So TTC (2013) Flourishing across Europe: application of a new conceptual framework for defining well-being. Soc Indic Res 110:837-61 
Jacobs AM (2016) Policy-making for the long term in advanced democracies. Annu Rev Polit Sci 19:433-54

Jenkins R, Baingana F, Ahmad R, McDaid D, Atun R (2011a) Health system challenges and solutions to improving mental health outcomes. Ment Health Fam Med 8:119-27

Jenkins R, Baingana F, Ahmad R, McDaid D, Atun R (2011b) International and national policy challenges in mental health. Ment Health Fam Med 8:101-14

Lawlor VM, Webb CA, Wiecki TV, et al. (2020) Dissecting the impact of depression on decision-making. Psychol Med 50:1613-1622. https://doi.org/ $10.1017 / \mathrm{s} 0033291719001570$.

Loesche D (2018) Lebanon still has the highest refugees density. Statista. https:// www.statista.com/chart/14323/countries-with-most-refugees-per-1000inhabitants/. Accessed 3 Dec 2019.

Krumpal I (2013) Determinants of social desirability bias in sensitive surveys: a literature review. Qual Quant 47:2025-2047

Medical Aid for Palestinians (2020) Barriers to the health and dignity of Palestinian refugees in Lebanon. https://www.map.org.uk/downloads/health-in-exilebarriers-to-the-health-and-dignity-of-palestinian-refugees-in-lebanon.pdf. Accessed 4 May 2020.

Miller KE, Rasmussen A (2017) The mental health of civilians displaced by armed conflict: an ecological model of refugee distress. Epidemiol Psychiatr Sci 26:129-38

Morina N, Akhtar A, Barth J, Schnyder U (2018) Psychiatric disorders in refugees and internally displaced persons after forced displacement: a systematic review. Front Psychiatry 9:433

Moya A (2018) Violence, psychological trauma, and risk attitudes: evidence from victims of violence in Colombia. J Dev Econ 131:15-27

Nair S, Howlett M (2017) Policy myopia as a source of policy failure: adaptation and policy learning under deep uncertainty. Policy Polit 45:103-18

Nardi PM (2019) Doing survey research: a guide to quantitative methods (\#4). Routledge, New York

Page L, Savage DA, Torgler B (2017) Variation in risk seeking behaviour following large losses: a natural experiment. Eur Econ Rev 71:121-31

Richman WL, Weisband S, Kiesler S, Drasgow F (1999) A meta-analytic study of social desirability distortion in computer-administered questionnaires, traditional questionnaires, and interviews. J Appl Psychol 84:754-775

Ruggeri K, Garcia-Garzon E, Maguire Á, Matz S, Huppert F. A.(2020) Well-being is more than happiness and life satisfaction: a multidimensional analysis of 21 countries. Health Qual Life Outcomes 18(1):1-16

Sangalang CC, Vang C (2017) Intergenerational trauma in refugee families: a systematic review. J Immigr Minor Heal 19:745-54

Santavirta T, Santavirta N, Gilman SE (2018) Association of the World War II finnish evacuation of children with psychiatric hospitalisation in the next generation. JAMA Psychiatry 75:21-27

Schildberg-Hörisch H (2018) Are risk preferences stable? J Econ Perspect 32:135-154

Thaler RH, Sunstein CR (2008) Nudge: improving decisions about health, wealth, and happiness. Yale University Press, New Haven

UN OCHA (2019) ReliefWeb crisis figures data. UN OCHA. https://data.humdata. org/dataset/0160cfdf-7dec-41a7-b0d2-8aa378f054d9. Accessed 2 Dec 2019.

WHO (2013) Mental health action plan 2013-2020. World Health Organization, Geneva

\section{Acknowledgements}

The authors would like to thank the executive board of the R4HC-MENA project for general comments in the development of this study. We also thank Rabih El Chammay, Nour Kik, and the Lebanese Ministry of Public Health for endorsing the method prior to data collection. We thank Prof. Simon Deakin and the Centre for Business Research for ethical oversight, and Zeina Afif for her feedback on the manuscript. Finally, we thank the Isaac Newton Trust (Trinity College, Cambridge) and Grand Challenges Canada for funding prior work that made this project possible. This publication is funded through the UK Research and Innovation GCRF Research for Health in Conflict (R4HC-MENA); developing capability, partnerships and research in the Middle and North Africa ES/P010962/1. The overall vision for the R4HCMENA partnership is to build sustainable research and policy capacity in the region to address major health challenges arising from conflict. R4HC-MENA activities aim to facilitate more effective translation of research into policy and deliver impact on both the research community and for patients and vulnerable populations. The study funder had no role in study design, and no involvement in data collection, data analysis, data interpretation, or writing the article. All authors had full access to all the data in the study and had final responsibility for the decision to submit for publication

\section{Competing interests}

KR reports funding from the Isaac Newton Trust, Trinity College, University of Cambridge.

\section{Additional information}

Supplementary information The online version contains supplementary material available at https://doi.org/10.1057/s41599-021-00784-z.

Correspondence and requests for materials should be addressed to K.R.

Reprints and permission information is available at http://www.nature.com/reprints

Publisher's note Springer Nature remains neutral with regard to jurisdictional claims in published maps and institutional affiliations.

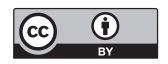

Open Access This article is licensed under a Creative Commons Attribution 4.0 International License, which permits use, sharing, adaptation, distribution and reproduction in any medium or format, as long as you give appropriate credit to the original author(s) and the source, provide a link to the Creative Commons license, and indicate if changes were made. The images or other third party material in this article are included in the article's Creative Commons license, unless indicated otherwise in a credit line to the material. If material is not included in the article's Creative Commons license and your intended use is not permitted by statutory regulation or exceeds the permitted use, you will need to obtain permission directly from the copyright holder. To view a copy of this license, visit http://creativecommons.org/ licenses/by/4.0/.

(C) The Author(s) 2021 